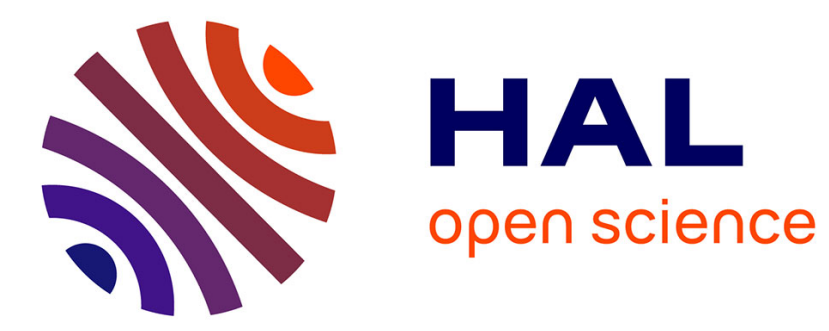

\title{
Motifs utilitaires et autonomie artistique: La forme architecturale et ses fonctions
}

Estelle Thibault

\section{To cite this version:}

Estelle Thibault. Motifs utilitaires et autonomie artistique: La forme architecturale et ses fonctions. Revue de Synthèse, 2012, 133 (4), pp.477-494. 10.1007/s11873-012-0200-9 . halshs-00772655

\section{HAL Id: halshs-00772655 \\ https://shs.hal.science/halshs-00772655}

Submitted on 14 Feb 2016

HAL is a multi-disciplinary open access archive for the deposit and dissemination of scientific research documents, whether they are published or not. The documents may come from teaching and research institutions in France or abroad, or from public or private research centers.
L'archive ouverte pluridisciplinaire HAL, est destinée au dépôt et à la diffusion de documents scientifiques de niveau recherche, publiés ou non, émanant des établissements d'enseignement et de recherche français ou étrangers, des laboratoires publics ou privés.

\section{(1) (1) $\$$}

Distributed under a Creative Commons Attribution - NonCommercial - NoDerivatives| 4.0 
Motifs utilitaires et autonomie artistique: la forme architecturale et ses fonctions

\section{Estelle Thibault}

Dans: Revue de synthèse, t. $133,6^{\mathrm{e}}$ série, $\mathrm{n}^{\circ} 4$ Esthétique de la technique, 2012, p. 477-494.

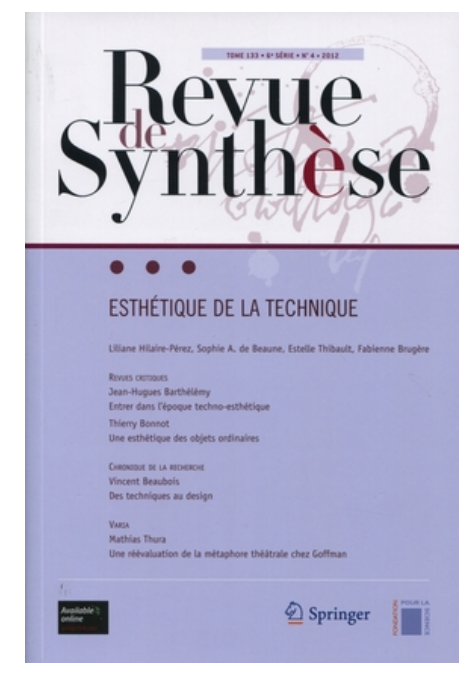

« Form follows function ». Derrière ce slogan attribué à l'architecte américain Louis Sullivan, se diffuse, au $x^{e}$ siècle, la thèse selon laquelle l'évaluation de la forme architecturale, loin d'être autonome, est intrinsèquement liée à la manière dont celle-ci satisfait à ses «fonctions". Dans le discours courant, la notion de «fonctionnalisme architectural » s'est propagée après la seconde guerre mondiale comme une catégorie à part entière, jusqu'à devenir un quasi-synonyme d'une architecture moderne qui subordonnerait les considérations artistiques à l'efficacité constructive, programmatique, utilitaire et sociale. Si le couple forme-fonction s'est ainsi placé au premier plan des débats architecturaux, le paradigme fonctionnaliste, bientôt questionné par la montée des critiques postmodernistes, a fait l'objet d'enquêtes visant à l'inscrire dans une histoire plus longue et à mieux en expliciter les fondements ${ }^{1}$. Les travaux récents révèlent surtout, au prisme d'une analyse des édifices comme des théories qui les accompagnent, l'écart entre le simplisme de l'acception courante du fonctionnalisme et la complexité des débats architecturaux.

\footnotetext{
${ }^{1}$ Parmi les travaux sur les origines historiques du fonctionnalisme et ses définitions voir notamment SFAELLOS, 1952 ; ZURCO, 1957; RICHARDS, 1958; JOEDICKE, 1971; GUILLERME, 1987, SCHNAIDT et GALLO, 1988.
} 
Certains chercheurs se sont attachés à déconstruire le mythe de la fonction dans l'avant-garde moderniste ${ }^{2}$, montrant que les déterminations utilitaires et constructives évacuent rarement les ambitions artistiques. D'autres, regrettant «l'infortune critique ${ }^{3}$ d'un fonctionnalisme souvent appauvri par ses détracteurs, ont identifié, derrière l'apparente homogénéité du terme, une pluralité de démarches guidant le travail de conception architecturale, déclinées à partir d'un vaste ensemble de notions : usage, utilitarisme, technique, science, rationalité, objectivité...

Ces approches sont confortées par une relecture des écrits fondateurs, restituant la richesse de concepts aplanis par la vulgarisation ou par la traduction. Le slogan moderniste form follows function, cité en ouverture, se révèle par exemple une interprétation très réductrice des propos de l'architecte américain, évacuant le vitalisme et les métaphores organiques dont ils étaient initialement chargés ${ }^{4}$. De même le terme «fonctionnalité », peu nuancé, a effacé les subtilités offertes par les substantifs allemands Sachlichkeit, mieux traduit par «objectivité », et Zweckmässigkeit, qui, dans le contexte architectural, concerne plus spécifiquement l'adaptation de l'édifice à ses usages, son efficacité pratique ${ }^{5}$.

Aux tentations d'une définition univoque et globalisante s'est ainsi substituée une relecture de l'évolution des rapports entre forme et fonctions. Ces relations se déclinent en de multiples formulations selon ce que les auteurs attachent à ces vocables $^{6}$. Le terme de fonction, tel qu'il s'est diffusé au $x x^{\mathrm{e}}$ siècle, désigne le plus souvent les activités spécifiques que l'édifice abrite. La fonction correspond au programme de l'édifice : elle renvoie à son usage, à son utilité sociale, censée s'extérioriser dans la forme donnée au bâtiment selon des degrés variés d'objectivité ou d'évocation symbolique. Ce rapport transparent entre forme et programme et la spécialisation des espaces qu'il sous-tend sont au cœur des critiques postmodernistes ${ }^{7}$. Mais cette acception de la fonction est assez récente, elle

\footnotetext{
${ }^{2}$ BENTON, 1990.

${ }^{3}$ REICHLIN, 1997.

${ }^{4}$ Voir à ce propos MANIERI-ELIA, 1996 ; VAN ZANTEN, WIT, 1986.

${ }^{5}$ Voir notamment BEHNE, 1926 et l'introduction à la traduction française de Der Moderne Zweckbau : STAVRINAKI, 2008.

${ }^{6}$ Voir FORTY, 2000.

${ }^{7}$ En particulier, l'architecte Robert Venturi propose de substituer au slogan Form follows function la formule Form evokes function, empruntée à Louis Kahn. Voir VENTURI [1966] 1999, p. 40.
} 
recouvre que l'on nommait auparavant la destination. Aux $X V I I I^{e}$ et $X I X{ }^{e}$ siècles, la notion de fonction s'applique plutôt aux éléments de l'édifice, elle désigne leur rôle dans la logique constructive, leur contribution à la solidité de l'ensemble. La discussion porte alors sur la manière dont la forme doit -ou non- dévoiler, refléter, donner à comprendre la réalité de ce système constructif. Ainsi l'expression des fonctions, qu'elles soient constructives ou utilitaires, se confronte de manière récurrente à d'autres enjeux: la charge symbolique de l'édifice, son régime d'expression, son action émotionnelle. Car la revendication du statut artistique de l'architecture implique, parallèlement à un désir croissant de rationalisation, l'affirmation d'une fonction esthétique, diversement conjuguée à ses autres rôles, et dont l'éventuelle autonomie est sans cesse discutée ${ }^{8}$.

Ces conceptions mouvantes des rapports entre forme et fonction sont tributaires des contextes intellectuels et culturels dans lesquels elles prennent place. En amont des interprétations modernistes, la France du XIX ${ }^{e}$ siècle apparaît comme un terrain particulièrement intéressant d'une redistribution hésitante des hiérarchies entre l'utile et le beau. La définition du beau comme idéal désintéressé se confronte à la montée de plusieurs idées, notamment celle d'un art utile, en phase avec les moyens techniques comme avec les nécessités de son temps ${ }^{9}$. Au milieu du siècle, les campagnes de valorisation des arts industriels ainsi que l'intérêt anthropologique croissant pour les arts dits secondaires motivent d'importants débats sur la hiérarchie des genres, sur l'origine des styles et sur la pertinence même de la notion de beau. L'aspiration à un art social, vecteur d'une harmonie populaire et susceptible de contribuer au bonheur du plus grand nombre ${ }^{10}$, tend également à réordonner ces hiérarchies, puisqu'en insistant sur la valeur émancipatrice du beau, elle confère à l'art un nouveau registre d'utilité.

Nous proposons de mettre en évidence certains de ces déplacements, en portant notre regard sur les échanges qui se tissent entre les réflexions des philosophes et celles des architectes. Les frontières entre théories de l'architecture et esthétique se révèlent poreuses, lorsque certains architectes, comme Viollet-le-

\footnotetext{
${ }^{8}$ Voir THIBAULT, 2010.

${ }^{9}$ Sur ces définitions de l'art utile et de son enseignement, voir LAURENT, 1998.

${ }^{10}$ Voir MCWILLIAM, [1993] 2007.
} 
Duc, contribuent aux débats sur l'orientation d'une science esthétique alors en cours de constitution disciplinaire. Réciproquement, les philosophes, occupés à définir le territoire scientifique de l'esthétique, rencontrent en l'architecture un objet dont l'impureté résiste aux modèles d'analyse applicables aux autres arts.

Si la distinction entre le registre d'une construction à caractère utilitaire et celui d'une architecture entendue comme art fonde, de manière durable, l'identité professionnelle des architectes, ceux-ci sont néanmoins parmi les premiers à chercher la voie d'une réconciliation possible entre le beau et l'utile, entre intention formelle, technique et usage. Ils insistent en effet sur le rôle social de l'art longtemps avant que celui-ci ne soit mis en exergue par les théoriciens de l'esthétique.

La Revue générale de l'architecture et des travaux publics, organe de presse dirigé par l'architecte César Daly, joue un rôle non négligeable dans ces évolutions, en diffusant un programme architectural résumé par la devise « le vrai, le beau, l'utile ». Substituer « l'utile » au « bien » dans une formule empruntée à Victor Cousin vise à réhabiliter une notion plutôt écartée dans les leçons sur le beau de ce dernier. Mais loin de vouloir réduire l'architecture à des considérations utilitaires, il s'agit au contraire d'élargir la définition de l'utilité. L'un des promoteurs de la formule, l'architecte Simon Claude Constant-Dufeux, précise que l'utilité rassemble trois notions - disposition, construction, décoration- ; qui recouvrent en somme l'ancienne triade vitruvienne -commodité, solidité, beauté. Ainsi anoblie, l'utilité, posée comme le «principe souverain» de l'architecture, ouvre un nouveau dialogue avec l'art, puisqu'elle ne concerne «pas seulement la satisfaction des besoins matériels, mais aussi la satisfaction des besoins d'un ordre plus élevé, je veux dire ceux de l'intelligence [...] l'utilité, prise dans le sens que je donne à ce mot, conduit à la grandeur morale et au beau ${ }^{11}$. Ces réflexions invitent déjà à penser l'utilité des formes architecturales selon ces différents registres : l'appropriation de l'édifice à ses usages, la rationalité constructive, l'efficacité expressive, l'ensemble étant investi d'une mission sociale. Elles contrastent avec le dédain avec lequel les philosophes contemporains abordent la question de l'utile.

\footnotetext{
${ }^{11}$ Constant-Dufeux, 1848, col. 445.
} 


\section{Des sciences du beau aux sciences de l'art. Hiérarchies du beau et de l'utile}

1.1. L'utilité, "vice incurable » de l'architecture?

Définie depuis Vitruve à l'articulation entre commodité, solidité et beauté, l'architecture est généralement considérée comme le parangon de l'art utile. $\mathrm{Ce}$ statut se confronte à l'idée kantienne du désintéressement du beau et vaut à l'architecture une place incertaine dans l'échelle des arts. La remise en cause de la supériorité du beau sur l'utile ne s'opère que lentement dans une philosophie française longtemps dominée par l'héritage de Victor Cousin. Autour de 1860, l'architecture trouve une place marginale dans les développements des philosophes de l'Académie, occupés à promouvoir une "science du beau " aux orientations spiritualistes $^{12}$. Les réflexions sur l'objet d'une telle science convergent vers une définition du «Beau » comme idéal détaché des nécessités matérielles. Une telle orientation n'est pas favorable à l'art de bâtir, dont le statut utilitaire justifie l'infériorité par rapport aux autres arts du dessin, sculpture et peinture. L'utilité est présentée comme le "vice incurable » de l'architecture, selon une expression proposée par le philosophe Édouard Antelme Chaignet : «Ce rapport à une fin, vice irrémédiable, incurable, de l'architecture, ne lui permet d'aspirer à obtenir, parmi les arts, que la place la plus humble. ${ }^{13}$ Ces sciences du beau qui paraissent autour de 1860 s'accordent également sur la méthode : une enquête philosophique et introspective sur le "sentiment du beau », faisant alterner la spéculation sur les principes avec la "vérification » par les grandes œuvres canoniques.

La Grammaire des arts du dessin $(1867)^{14}$ du théoricien de l'art Charles Blanc, bientôt titulaire de la première chaire d'esthétique au Collège de France, prolonge l'argumentaire. L'architecture ne pourrait être considérée comme un art que dans la mesure où ses œuvres s'émancipent des nécessités physiques pour devenir de « pures créations de l'esprit ». II s'agit d'affirmer, en somme, la différence entre l'art

\footnotetext{
12 En 1857, la section de philosophie de Académie des sciences morales et politiques lance un concours pour la définition d'une "science du beau ». Parmi les ouvrages lauréats et mentionnés voir LEVEQUE, 1861, CHAIGNET, 1860.

${ }^{13}$ ChAIGNET, 1860, p. 514. L'expression est discutée par BLANC, [1867] 1880, p. 105-106 ; SouRIAU, 1904, p. 192.

${ }^{14}$ BLANC, [1867] 1880.
} 
monumental, caractérisé par son indépendance, et la simple construction. Une telle position est cohérente avec le présupposé d'une incompatibilité entre l'utile et le beau :

L'utile est le domaine de l'industriel ; le beau est l'apanage de l'artiste ; on admire les créations de l'art, on consomme les produits de l'industrie. Dès que la beauté n'est pas la première qualité d'un objet, cet objet n'est point une œuvre d'art. Un meuble utile peut avoir une certaine beauté, mais il n'est pas beau en lui-même et par essence ; il n'est qu'embelli. Quand l'utile et le beau sont réunis dans une même chose, il arrive souvent que la beauté semble en interdire l'usage, et, si elle l'emporte, l'objet devient alors inutile ${ }^{15}$.

Blanc réfute radicalement idée d'une origine «matérielle » de l'art monumental :

En remontant aux époques primitives, on aperçoit deux genres de construction bien distincts, l'un pour le corps, l'autre pour l'âme. À côté de l'industrie qui bâtit la demeure où l'homme renferme sa famille et sa personne, l'architecture édifie le monument qui doit résumer les croyances et les aspirations du peuple entier ${ }^{16}$.

Les positions de Charles Blanc doivent être comprises à l'aune de son engagement pour le maintien des divisions entre les arts ainsi que des hiérarchies entre arts majeurs et mineurs. Sa Grammaire se fonde sur une répartition des arts en «genres » distincts : s'il existe des principes généraux, valables pour l'ensemble des «arts du dessin », ceux-ci sont fondamentalement différents des lois qui régissent les «arts décoratifs », qui font l'objet d'un volume séparé ${ }^{17}$. La partie consacrée à l'architecture écarte toutes considérations utilitaires et constructives, non pertinentes puisqu'elles ne concernent pas l'architecture comme art. S'il s'agit de s'interroger sur les origines de l'art pour en exposer les fondements, l'enquête est toute spéculative et non historique, Blanc affirmant toute sa méfiance vis-à-vis d'approches archéologiques qui font primer les faits sur les principes.

Lorsqu'il occupe, en 1863, la première chaire d'esthétique et d'histoire de l'art à l'École des beaux-arts, l'architecte Eugène Emmanuel Viollet-le-Duc adopte un tout

\footnotetext{
15 Ibidem, p. 15.

${ }^{16}$ BLANC, 1867 (1880), p. 55.

17 BLANC, 1882.
} 
autre angle de vue ${ }^{18}$. L'argument en est bien connu: il s'agit d'expliquer le développement des arts en centrant le regard sur les conditions de leur apparition, conditions à la fois matérielles, techniques et morales. La méthode, clairement historique, propose de lire les expressions artistiques dans leur milieu. Elle implique de minimiser la division des arts telle que Blanc la formulait pour repérer, dans l'ensemble des productions techniques et artistiques d'une civilisation, les traits stylistiques qui lui sont propres. La séparation entre arts industriels et arts majeurs ainsi que la distance entre l'utile et le beau se voient d'autant plus remises en cause que l'origine des arts est située dans la satisfaction des besoins primitifs ${ }^{19}$. Ainsi par exemple, à l'irréductibilité entre construction ordinaire et architecture monumentale défendue par Blanc, Viollet-le-Duc oppose, sur des bases archéologiques, la continuité entre les dispositions des habitations primitives d'un peuple et son architecture religieuse et civile. L'architecture est le point focal d'une démonstration plus globale concernant la rationalité de l'activité artistique, dont il s'agit d'expliquer la genèse et le développement, en insistant sur les déterminismes matériels de la forme. L'histoire de l'architecture, désormais inscrite dans une histoire plus vaste des artefacts, propose ainsi de nouveaux paradigmes pour une esthétique progressivement définie comme une science de l'homme et de la société.

\subsection{Perspectives évolutionnistes : de l'utile vers le beau}

Dans la philosophie esthétique des décennies suivantes, les hiérarchies entre le beau et l'utile s'infléchissent sous l'influence de ces nouveaux axes historiographiques et d'une pensée évolutionniste qui enquête sur les origines de l'art. Certes, Herbert Spencer décrit encore l'activité artistique comme une pulsion dérivée du jeu, fondamentalement définie par sa gratuité et son indépendance vis-àvis des besoins fondamentaux. Elle résulte, explique-t-il, de la dépense d'un surplus d'énergie, non mobilisée dans la conservation de la vie. Si, dans un essai intitulé «L'Utile et le beau», Spencer défend fermement leur antinomie, il suggère

\footnotetext{
${ }^{18}$ Voir ses leçons : VIOLLET-LE-Duc [1864], 1994.

19 "L'art, c'est l'expression sensible, pour tous, d'un besoin satisfait », VIOLLET-LE-DUC, 1863, vol. 1, p. 24.
} 
cependant un lien de continuité de l'un et l'autre. Le beau naîtrait de l'utile, au moment où celui-ci perd son usage :

Ce que la nature a jadis créé afin de répondre à un usage, elle s'en sert comme d'ornement [...] la structure d'un coquillage de mer, dans lequel les organes qui, à une certaine période, ont été la bouche, se trouvent à une autre période de sa croissance rejetés en arrière, et deviennent des nœuds et des épines dont le coquillage est paré ${ }^{20}$.

Des ornements de la nature, traces émouvantes d'anciens organes devenus inutiles, le raisonnement s'étend à la beauté des ruines, souvenirs d'une ancienne activité :

Ce qui avait été bâti pour être un abri ou une sauvegarde, ce qui, dans ces temps anciens, avait, dans l'économie de la société, un rôle important, est maintenant devenu pur ornement ${ }^{21}$.

La perspective évolutionniste suggère cependant des pistes plus concrètes, lorsqu'elle se conjugue à l'ouverture de l'esthétique aux apports des sciences humaines et sociales. L'Esthétique d'Eugène Véron prend toutes ses distances vis à vis de la thèse d'une origine « idéale » de l'art et contribue à introduire de nouveaux modèles pour son étude, cherchant ses méthodes du côté de la psychophysiologie et de l'anthropologie ${ }^{22}$. En alternative aux enquêtes métaphysiques sur les principes du beau, Véron prône un regard historique sur la naissance et le développement des pratiques artistiques. Le geste esthétique s'imprimerait, dès la préhistoire, aux armes et aux outils ; des ornements qui parent les premiers instruments dériveraient peu à peu les créations artistiques. II existerait un progrès continu, depuis les œuvres décoratives de l'artisanat primitif vers celles dites expressives de l'art le plus élevé. À la notion même de beau, assimilée à l'agréable et au décoratif, Véron substitue, pour l'évaluation des œuvres, celle de perfection, considérant qu'un même « instinct du mieux » guide les productions humaines. La séparation entre les œuvres de l'industrie et celles de l'art tend alors à s'estomper, au profit d'un modèle critique capable de les embrasser ensemble. L'architecture acquiert, dans une telle approche, un statut d'objet d'étude particulièrement démonstratif, dont les critères

\footnotetext{
${ }^{20}$ SPENCER, 1877, p. 253.

${ }^{21}$ Idem, p. 254.

${ }^{22}$ VERON, 1878. Sur cet auteur voir COLRAT, 2008.
} 
d'évaluation pourraient s'étendre aux autres arts. S'il adopte une définition de l'art empruntée à l'architecte Eugène Emmanuel Viollet-le-Duc - «pour l'architecte, l'art, c'est l'expression d'un besoin satisfait ${ }^{23}$ - il lui emprunte également ses perspectives méthodologiques, croisant les développements techniques avec les évolutions formelles, soulignant les interactions entre les dispositifs statiques et l'expression artistique. L'architecture, en prise avec les réalités matérielles comme avec les nécessités symboliques, fournit alors un modèle pour l'analyse des autres arts, dont les contingences ne sont pas négligées.

\subsection{Esthétiques sociologiques et « beauté rationnelle »}

L'élargissement du champ disciplinaire de l'esthétique motive ainsi un renversement de la hiérarchie entre l'utile et le beau ; renversement conforté, sur la fin du siècle, par les premières esthétiques sociologiques. Jean-Marie Guyau soutient à son tour l'idée d'une continuité entre les œuvres de l'industrie et celles de l'art, liées historiquement dans un progrès du sens du beau et guidées par un même souci de perfection. II existe sans doute encore un écart entre "la beauté très restreinte de l'utile ", liée à la satisfaction que procure l'adaptation parfaite d'un objet à son usage, et " tous les autres genres plus larges de libre beauté ${ }^{24}$; néanmoins l'utile et le beau convergent vers un même but social et humain. L'architecture apparaît alors comme l'art social par excellence, vecteur d'une sympathie collective. Dans les Problèmes de l'esthétique contemporaine (1884) comme dans L'art au point de vue sociologique (1889), la remise en cause des frontières entre l'utile et le beau renvoie clairement à celle des hiérarchies sociales qui les fondaient. Guyau soutient en effet que le sentiment du beau du paysan ou de l'ouvrier, face au produit d'un travail satisfaisant, n'est pas fondamentalement différent de celui de l'amateur d'art.

Dans La Beauté rationnelle (1904) de Paul Souriau, l'exemple de l'architecture appuie le chapitre consacré à la "valeur esthétique de la finalité ${ }^{25}$, jusqu'à prendre à rebours la proposition de Chaignet : «nous ne la défendrons pas d'être un art

\footnotetext{
${ }^{23}$ VIOLLET-LE-DUC, 1863, vol. 1, p. 24, cité par VERON, 1878, p. 189.

${ }^{24}$ GUYAU, 1889, p. 12-13. Voir aussi GuYAU, 1884, notamment ch. "De l'antagonisme de l'art et de l'industrie moderne », p. 115-122.

${ }^{25}$ SOURIAU, 1904, p. 198-212.
} 
appliqué, nous l'en glorifierons ${ }^{26}$. L'écart se réduit entre l'architecture, art utile et rationnel, et les autres arts, dont il démontre qu'il ne sont pas moins inférés à des nécessités techniques, symboliques et sociales. Aux antagonismes supposés entre l'utile et le beau, Souriau substitue ceux qui opposent les diverses fins : " conflit entre nos fins et celles d'autrui, conflit entre une fin inférieure et une fin supérieure ${ }^{27}$. Souriau suggère alors d'analyser les finalités de l'art et d'évaluer l'œuvre selon la manière dont elle satisfait les différentes attentes ; la tâche d'une véritable science esthétique serait d'appréhender ces conflits et d'en hiérarchiser les enjeux.

Entre philosophie de l'art et théories de l'architecture, les textes évoqués ici montrent un double mouvement. L'art de bâtir y assume plus franchement sa dimension technique et sa vocation sociale, revalorisées au prisme d'une définition du beau comme perfection et d'une lecture évolutive de l'histoire. D'autre part, l'architecture conquiert une pertinence nouvelle pour une discipline esthétique dont le périmètre se déplace, de la philosophie spiritualiste vers les sciences humaines et sociales. Elle devient un objet d'étude particulièrement intéressant pour comprendre le rôle social de l'art et ses déterminations techniques, à l'encontre de l'idée d'un pur désintéressement et d'une liberté de la création artistique.

\section{Les formes architectoniques: vérité constructive et expression artistique}

Confrontés plus concrètement, dans leur pratique, aux interactions entre esthétique, technique et usage, les architectes ont pointé très tôt ces interférences entre les registres d'utilité, comme le montre la posture de Constant-Dufeux citée précédemment. Sur la séquence qui sépare les avis respectifs de Chaignet et de Souriau, ils affinent leur analyse sur les différents niveaux de détermination de la forme et précisent le lieu éventuel de l'autonomie artistique.

Les interrogations sur les fondements du vocabulaire architectural classique illustrent de manière remarquable les déplacements qui s'opèrent. Cohérente avec les valeurs philosophiques portée par l'Académie, la thèse traditionnelle sur l'origine

\footnotetext{
${ }^{26}$ Idem, p. 191.

${ }^{27}$ Idem, p. 212.
} 
des ordres grecs postule l'autonomie de l'expression artistique. Manifestation d'un idéal, les formes de l'art grec auraient leur raison propre. Tout en s'inspirant parfois de motifs constructifs -selon la théorie qui fait dériver l'ordre dorique de temples primitivement édifiés en bois- les formes architectoniques les idéalisent, elles trouvent d'autres modèles dans le corps humain ou dans les créations naturelles et expriment de fait un ordre esthétique et symbolique supérieur à celui des exigences techniques. Au XIX ${ }^{e}$ siècle les débats sur l'origine et l'évolution des styles, depuis leurs manifestations primitives dans les industries d'art vers les formes plus évoluées de l'art monumental, ouvrent à d'autres conceptions des relations entre détermination matérielle et expression artistique.

\subsection{La thèse de la vérité constructive : déterminations fonctionnelles}

Une première ligne de pensée est ouverte par Viollet-le-Duc, lorsque celui-ci soutient l'hypothèse d'une origine toute fonctionnelle de la forme. Sur ce point, la fécondité des emprunts conceptuels aux sciences naturelles a souvent été soulignée, nous n'en rappellerons que les aspects qui concernent le rapport entre forme et fonction ${ }^{28}$. Les travaux de Georges Cuvier sur l'organisation animale offrent de puissantes analogies qui parcourent l'œuvre théorique de Viollet-le-Duc. Derrière le concept de «conditions d'existence », Cuvier défend la thèse selon laquelle l'environnement détermine complètement la morphologie animale. Les animaux de toutes les espèces, dans leur diversité, sont analysés comme autant de systèmes logiques, unitaires et rationnels, surdéterminés par leurs conditions de vie dans un environnement spécifique et composé d'organes répondant chacun à des fonctions précises. De cette parfaite adéquation dépend leur survie. Dans les écrits de Violletle-Duc, la métaphore de l'édifice comme organisme propose de penser la forme de l'élément constructif en relation avec sa fonction dans un système global qui répond à des conditions extérieures -matériaux, savoirs techniques, climat, mœurs, contexte politique, social et religieux- hors desquelles il perd toute validité. Ainsi du temple dorique, considéré comme exemplaire d'un art autochtone, né du sol de la Grèce, des matériaux et des techniques constructives disponibles, et à propos

\footnotetext{
${ }^{28}$ Voir notamment VAN ECK, 1994, qui souligne également la force de ces analogies chez d'autres auteurs tels que Léonce Reynaud; BRESSANI, 1996 ; BARIDON, 2001.
} 
duquel il réfute radicalement l'hypothèse selon laquelle sa forme architectonique serait la transposition en pierre d'un dispositif primitivement conçu en bois.

Car le principe d'un strict déterminisme est posé, dans les Entretiens sur l'architecture, comme une norme d'évaluation de la qualité architecturale au vu de ses conditions d'existence. La valeur esthétique étant indexée sur l'efficacité fonctionnelle, l'autonomie artistique de la forme se réduit, du moins en théorie. L'impératif esthétique n'est cependant pas évacué et la beauté cherche aussi ses raisons, notamment du côté des lois de l'optique qui déterminent les proportions à privilégier. Quant à la décoration, elle trouve également des justifications du côté de nécessités symboliques. Loin de se développer librement, l'ornementation doit être l'expression vraie des besoins. Elle résulte de diverses contingences comme l'appropriation aux usages ou au climat ; elle répond à des exigences symboliques, empruntant à l'univers des créations naturelles, flore ou faune, mais elle est en premier lieu dépendante de la logique structurelle. Si Viollet-le-Duc reconnaît la légitimité de l'ornement, le principe de vérité s'oppose à des écarts trop importants entre apparence et construction, sur lesquels l'architecte porte un jugement moral. L'ornement ne peut entraver la lisibilité d'une structure qu'il doit, au contraire, rendre plus explicite. II s'agit ainsi de refuser fermement la reconduction d'écritures ornementales superficielles qui, héritées de traditions architectoniques antérieures, ont perdu leurs bases matérielles et leur légitimité constructive.

\subsection{Louis Cloquet : formes de convenance, de structure et d'expression}

Dans la lignée de ces propos, l'architecte et ingénieur belge Louis Cloquet, nettement moins connu, s'interroge à son tour sur les différents niveaux de détermination des formes. Lecteur attentif de ses prédécesseurs et contemporains, architectes ou esthéticiens, Cloquet s'aventure à deux reprises sur le terrain de l'esthétique appliquée à l'architecture. Son Essai sur les principes du beau en architecture paru en $1894^{29}$ est suivi l'année suivant par une Esthétique architecturale. Essai de classification et d'appréciation des formes ${ }^{30}$, tous deux seront ensuite intégrés dans le cinquième volume, consacré à l'esthétique et à la

\footnotetext{
${ }^{29}$ Cloquet, 1894.

${ }^{30}$ CLOQUET, 1895.
} 
composition, de son Traité d'architecture ${ }^{31}$. Ces textes veulent ajuster ses convictions d'architecte, à savoir, son engagement pour un rationalisme constructif inspiré de Viollet-le-Duc, avec une définition du beau plutôt redevable de la philosophie spiritualiste.

L'entreprise est motivée par un désarroi face à l'éclectisme stylistique contemporain, appelant une clarification des critères d'évaluation applicables à l'art de bâtir. Afin d'appréhender la diversité des systèmes architecturaux en présence, l'architecte propose une classification dont l'originalité est de ne procéder ni par type de géométrie, ni par familles constructives ou techniques, mais d'organiser une typologie selon les registres de détermination des formes. La distinction majeure qu'il opère entre les «formes de convenance», les «formes de structure » et les «formes d'expression» découle de l'actualisation de la triade vitruvienne. Les premières « ont pour but de satisfaire à la première des conditions de l'art, l'utilité et la convenance »; dictées par l'appropriation aux « besoins » et à la destination, elles règlent les grandes lignes de disposition générale et s'appliquent en premier lieu au dessin du plan des édifices. Les deuxièmes « résultent de la structure et par suite répondent à une autre condition indispensable, la solidité »; elles dépendent des «moyens d'exécution », déterminent l'essentiel « des membres et des organes de l'édifice » ${ }^{32}$. Quant aux formes d'expression, elles sont conçues « dans le but spécial d'impressionner le spectateur », elles règlent les traits secondaires et accessoires. L'essai de classification propose donc de distinguer les motivations dont résultent les formes, afin d'évaluer leur efficacité dans chacun de ces registres. Notons que si les trois ne sont pas a priori hiérarchisés, les deux derniers font l'objet d'un développement bien plus important, car c'est bien au rapport entre structure et expression que Cloquet souhaite s'affronter.

Sitôt posées, ces catégories se divisent à nouveau en sous-groupes qui dénotent des interférences entre les contingences pratiques, les solutions constructives et le registre de l'esthétique. Le terme même de convenance, traditionnellement préféré dans les traités d'architecture à celui d'utilité, confronte à la stricte appropriation aux usages les conventions de la représentation symbolique.

\footnotetext{
${ }^{31}$ CLOQUET, 1913. Ce Traité rassemble le contenu de son enseignement à l'université de Gand.

${ }^{32}$ Cloquet, 1913, p. 26.
} 
La convenance «matérielle » se double ainsi de la convenance «morale », qui détermine, par exemple, «l'élancement des tours d'églises [...] indépendamment de leurs dimensions utiles ${ }^{33}$. Mais les interférences touchent surtout les catégories de la structure et de l'expression. Concernant les «formes de structure», Cloquet distingue les formes de structure « réelles », qui reflètent les moyens effectifs de la construction, de celles «fictives » qui indiquent « non le moyen employé, mais un moyen simulé ${ }^{34}$, enfin des formes de structure «transposées », «fruits d'une routine obstinée et aveugle $»^{35}$, qui perpétuent des formes liées à d'autres matériaux et techniques. Partisan de la rationalité constructive, l'auteur condamne ces dernières et leur préfère les formes « réelles »; il est cependant moins radical à l'encontre des «formes de structure fictives », considérant qu'elles peuvent aider à « idéaliser » l'appareil constructif, dans le cas d'assemblages peu hiérarchisés ou peu lisibles. Elles viennent en quelque sorte «accentuer la fonction par un redoublement de la forme » et rentrent alors, conclut-il, « dans la catégorie des formes d'expression ${ }^{36}$. Dans cette troisième catégorie figurent les formes « décoratives». Elles peuvent être «d'invention», purement géométriques, ou « d'imitation », lorsqu'elles trouvent leur inspiration dans la nature ou du côté de l'industrie humaine. Elles enrichissent alors l'édifice d'un discours iconographique. Néanmoins leur domaine doit rester soigneusement circonscrit à une ornementation raisonnée, «qui n'occupe que certaines parties choisies et caractéristiques de l'architecture », soulignant et accompagnant la structure. Les «formes d'expression » doivent ainsi rester indexées sur la construction, sous peine de supplanter les formes de structure. En leur fournissant un commentaire éloquent, elles permettent néanmoins de poétiser ces dernières, « brutales et prosaïques » 37 . Elles confèrent alors à l'édifice sa signification monumentale.

La typologie proposée par Cloquet appuie ainsi un projet de rationalisation. II prône un certain réalisme mais, loin de débarrasser la forme de ses attendus esthétiques, il en reconnaît aussi les nécessités symboliques. On perçoit les

\footnotetext{
33 Idem, p. 14.

34 Idem, p. 18.

${ }^{35}$ Idem, p. 82.

${ }^{36}$ Idem, p. 80.

37 Idem, p. 46.
} 
ambiguïtés de ce fonctionnalisme structurel inspiré de Viollet-le-Duc. Le principe moral de la vérité constructive ouvre la voie d'une objectivité architectonique plus radicale, subordonnant plus complètement la forme à la résolution ingénieuse des questions utilitaires et structurelles, sans encore l'explorer entièrement. La reconnaissance d'une fonction expressive de l'architecture oppose une résistance à l'idée d'une transparence totale entre la forme et ses autres fonctions.

\section{3. des motifs utilitaires aux formes de l'art monumental}

\subsection{Origine matérielle et transposition artistique}

D'autres voies sont explorées par les architectes pour concilier un regard positif sur les exigences fonctionnelles de l'architecture avec son statut artistique. Le problème de l'origine, matérielle ou idéale, des formes architectoniques, trouve alors d'autres analogies fécondes du côté des sciences naturelles. À la thèse de Cuvier, selon lequel la fonction dicte la forme de l'organe, Geoffroy Saint-Hilaire substitue une autre hypothèse, où la diversité animale dérive d'un seul plan d'organisation : une forme « originaire » de la création est ensuite déclinée à l'infini, le schéma formel précédant la fonction ensuite assumée par l'organe. Les théories de l'évolution, en posant le problème de la transformation de l'organisme face à l'évolution de son cadre de vie, invitent également à porter un autre regard sur la généalogie des formes. II faudrait alors saisir les interactions entre d'un côté, ce qui relèverait d'un principe formel originaire -sinon idéal-, de l'autre, les facteurs conjoncturels qui infléchissent celui-ci. Parallèlement, les sciences du langage et les découvertes archéologiques éclairent, au prisme de l'anthropologie naissante et d'une histoire générale de l'humanité, certains processus de transformation et d'hybridation des langues et des savoirs techniques.

Si les écrits français sur l'art de bâtir se montrent attentifs à ces recherches, L'architecte allemand Gottfried Semper est sans doute celui qui se saisit au plus près de leurs implications pour l'art de bâtir, avant qu'un français, Charles Chipiez, ne développe à son tour des hypothèses analogues. L'histoire comparée des styles proposée par Semper dans Der Stil in den technischen und tektonischen Künsten oder Praktische Ästhetik (1860-63) ouvre à une étude croisée des arts et des techniques, portant plus spécifiquement l'attention sur les interactions entre 
processus de fabrication, usages et expression formelle ${ }^{38}$. En ce sens, il offre une contribution méthodologique qui sera rapidement considérée comme fondatrice des approches anthropologiques de l'art ${ }^{39}$. Rappelons que Der Stil élabore une typologie des productions techniques et artistiques à partir de quatre «motifs » originaires, initialement liés à des matériaux et à des techniques primitives : la céramique (les arts du feu), la stéréotomie (l'art de la pierre), la tectonique (la charpenterie) et les arts textiles ${ }^{40}$. Le terme motif (Motiv) doit être compris dans son sens double, à la fois forme et cause. À la diffusion de ces motifs, des techniques vers l'art, se superpose leur transformation, d'un matériau à l'autre, ainsi que leur diffusion d'une civilisation à une autre. Dans ces processus, les changements de matériaux (Stoffwechse), loin d'impliquer un renouvellement radical, se confrontent à la permanence de caractéristiques formelles antérieures, lorsque celles-ci ont conquis une valeur symbolique forte. Comme l'a montré Harry F. Mallgrave, Semper affirme en outre l'existence d'une autonomie de la forme artistique par rapport à la résolution des problèmes techniques, matériels et utilitaires ${ }^{41}$. L'activité artistique s'exerce sur un registre scénographique, fondamentalement émancipé d'une réalité qu'il s'agit de travestir. Ainsi, l'architecture et les arts appliqués sont les produits conjugués de facteurs internes et externes: l'évolution de leur expression artistique et ornementale, investie de significations rituelles et culturelles, compose, sur des temporalités différenciées, avec celle des usages et des modes constructifs.

\subsection{Charles Chipiez : des arts secondaires vers l'autonomie esthétique}

La réception de l'œuvre encyclopédique de Gottfried Semper en France reste aujourd'hui encore mal évaluée ${ }^{42}$. Elle n'a certes pas d'équivalent français, néanmoins, plus modestement, l'ouvrage que l'architecte Charles Chipiez consacre, en 1876, à l'Histoire critique des origines et de la formation des ordres grecs

\footnotetext{
${ }^{38}$ Voir à ce propos MALLGRAVE, 1996, notamment ch. 4, «The Zurich Years : 1855-1869 », p. 229308, et SOULILLOU, 2007.

${ }^{39}$ Notamment dans le panorama des tendances de l'esthétique allemande proposé par le philosophe Victor Basch. Voir BASCH, 1920.

${ }^{40}$ SeMPER, 1863.

${ }^{41}$ MaLLGRAVE, 1996, notamment p. 277-302.

${ }^{42}$ Concernant la réception de Semper en France voir BerRY, 1993; Mallgrave, 1996; GargianI, 1997; BOURLIER, 2006.
} 
emprunte certaines des voies explorées par Semper ${ }^{43}$. Méconnu, ce livre ouvre des perspectives nouvelles, lorsqu'il propose d'interroger la genèse des formes architectoniques dorique, ionique et corinthienne dans le cadre d'un processus évolutif double. D'une part, une "théorie de la filiation des formes " ${ }^{44}$ va à l'encontre de l'idée d'un art grec autochtone. Elle s'appuie sur les découvertes archéologiques des expéditions scientifiques d'Égypte, d'Assyrie, de Perse et de Phénicie pour montrer comment les formes canoniques de la Grèce résultent d'influences et d'hybridations multiples. D'autre part, l'architecte, réfutant l'idée de formes imitatives qui trouveraient leur modèles dans les créations naturelles, veut suivre la naissance et le développement de motifs qui prennent leur origine dans les techniques des arts secondaires, pour se transférer ensuite vers l'art monumental. Car l'art naîtrait dans les techniques primitives; en effet, si, dans la période "anté-architectonique " l'édification accuse encore "toutes les vulgarités» des nécessités les plus élémentaires auxquelles elle répond platement, se constate déjà, dans les premières industries, un "amour de la parure ", expression embryonnaire d'un instinct artistique bientôt mieux déployé dans l'architecture ${ }^{45}$.

L'étude des «formes primordiales 》 de colonnes caractéristiques des diverses civilisations permet d'évaluer le rôle des différents facteurs qui en déterminent les caractéristiques : les proportions, les articulations entre chacun des éléments -base, fût, chapiteau, entablement...- et l'appareil ornemental. II s'agit de faire la part des choses entre ce qu'impose la destination, ce qui relève des matériaux, de leur mise en œuvre et des impératifs liés à la solidité, mais aussi ce qui procède soit de la reprise de dispositifs antérieurs, soit encore de motifs diffusés par les arts secondaires. Car loin de faire de la forme le produit des exigences conjuguées de l'utilité et de la construction, Chipiez fait entrer en ligne de compte le poids de l'attachement à certaines formes. Ainsi l'autonomie de l'expression artistique se mesure aux permanences formelles qui subsistent, d'une civilisation à l'autre, en dépit des mutations techniques. II s'agit donc de comprendre l'interaction des

\footnotetext{
${ }^{43}$ CHIPIEZ, 1876.

${ }^{44}$ Idem, p. IV.

${ }^{45}$ Idem, p. 343-344.
} 
facteurs utilitaires, matériels et techniques, tout en reconnaissant l'existence d'un « ordre expressif supérieur à celui des exigences matérielles » 46 .

Un rôle prioritaire est accordé aux matériaux dans la naissance des types formels. Ainsi l'emploi du bois, en Asie mineure, aurait conduit à fixer un type canonique de temple aux colonnes sveltes et aux larges entrecolonnements, dessinant un rapport entre pleins et vides longtemps adopté y compris dans des temples lapidaires, avant d'être concurrencé par un type égyptien aux piles épaisses et rapprochées, mieux accordé avec la logique inhérente à la pierre. La nature du matériau n'est donc pas indifférente aux proportions générales. Mais Chipiez insiste pour distinguer les nécessités de la construction, plutôt minorées, d'un autre type d'influence qu'exercent les « matériaux »" C'est dans le « mode de travail », c'està-dire dans les procédés de façonnage du matériau que s'inventeraient les premiers motifs.

L'architecte met alors l'accent sur les processus de diffusion de motifs découlant de l'usage de telle ou telle matière :

La prédominance que, suivant les époques, l'architecte accorde dans les édifices à certains matériaux, imprime un caractère particulier aux formes monumentales. [...] Quand le marbre est à peu près exclusivement employé, on doit s'attendre à ce qu'il impose, dans une certaine mesure, aux matériaux accessoires, les caractères qui le distinguent en propre ${ }^{48}$.

Cette contamination de motifs d'abord liés aux matériaux privilégiés est particulièrement lisible à partir de l'étude des «arts somptuaires de l'Orient ${ }^{49}$. Chipiez attire l'attention sur l'influence qu'exercent des motifs ornementaux primitifs résultant des techniques appliquées aux matériaux.

II explique que les thèmes formels inhérents au travail du métal et de la céramique, couramment employés dans les objets utilitaires, armes, outils, meubles et autres, connaissent une diffusion importante. D'une région à l'autre, les conquêtes transportent ces ustensiles décorés, favorisant la propagation de leurs motifs, dont s'empare rapidement l'art monumental. L'emploi du métal, utilisé dans ces objets et

\footnotetext{
${ }^{46}$ Idem, p. III.

47 «Nous disons l'importance des matériaux et non l'importance de la construction », idem, p. 348.

${ }^{48}$ Idem, p. 153.

${ }^{49}$ Idem, p. 153-167.
} 
dans des revêtements appliqués aux édifices, fournit alors des motifs compatibles avec l'évolution matérielle future du temple en pierre. Ainsi, par exemple, certaines « formes affectées à l'airain », comme les volutes, pourront bientôt donner « la forme élémentaire du chapiteau ionique ${ }^{50}$.

Sans discuter l'exactitude des hypothèses archéologiques de Chipiez, limitonsnous à relever l'intérêt du schéma évolutif qui va des formes décorées de l'industrie, liées aux matériaux et aux techniques qui les façonnent, vers celles, plus élevées, de l'architecture. On voit les techniques utilitaires progresser vers l'art monumental en libérant peu à peu l'apparence de ses impératifs initiaux. La forme architectonique prend sa source dans les traditions matérielles qui lui sont adjacentes; elle s'émancipe ensuite de ces exigences premières pour suivre une évolution plus indépendante, guidée par ses propres ambitions.

Ces théories n'esquissent que quelques unes des voies explorées par les architectes pour tenter d'appréhender les continuités entre l'industrie et l'art, tout en maintenant une distinction entre le niveau prosaïque de la construction ordinaire et celui plus élevé de l'art monumental. Elles gagneraient naturellement à être restituées dans un panorama plus vaste, comparées notamment aux développements germaniques contemporains sur le «réalisme » architectural $\left.\right|^{51}$. L'intérêt de ces tentatives est de penser la forme architectonique comme le facteur conjugué de différentes déterminations; d'affronter la complexité des interactions entre les bases matérielles et la sphère esthétique. Non seulement les dimensions utilitaires et constructives ne sont plus perçues comme contradictoires avec les finalités expressives, mais de nouvelles articulations se nouent entre ces registres. L'idée d'un simple dialogue, plus ou moins étroit, entre un cœur structurel et un vêtement artistique ne rend que partiellement compte d'interférences plus subtiles révélées par une lecture évolutive des styles. Lire l'art monumental comme la transposition, au prisme d'intentions esthétiques, de motifs techniques originaires, permet d'envisager autrement le lieu de l'autonomie artistique ainsi que la portée symbolique, culturelle et mémorielle des formes architectoniques.

\footnotetext{
${ }^{50}$ Idem, p. 160-161.

${ }^{51}$ Pour un panorama de ces débats sur le terrain germanique voir BERRY, 1993 ; MALLGRAVE, 1993.
} 


\section{Références bibliographiques}

BARIDON (Laurent), 2001, « Anatomie comparée et pensée évolutionniste dans la théorie et la pratique architecturale de Viollet-le-Duc », dans CENTRE D'ETUDES FOREZIENNES, L'architecture, les sciences et la culture de l'histoire au XIX siècle, p. 73-82.

BASCH (Victor), 1920, "L'esthétique nouvelle et la science de l'art », L'Esprit Nouveau, n 1, octobre, p. 5-12 et $\mathrm{n}^{\circ} 2$, novembre, p. 119-130.

BeHNE (Adolf), 1926, Der moderne Zweckbau, Berlin, Drei Masken Verlag (tr. fr. 2008, La Construction fonctionnelle moderne, Paris, Éditions de la Villette).

Benton (Tim), 1990, « The Myth of Function », dans Greenhalgh (Paul) (dir.), Modernism in Design, Londres, Reaktion Books, p. 41-53.

BERRY (James Duncan), 1993, "From Historicism to Architectural Realism : On Some of Wagner's Sources ", dans MallgRAVE (Harry F.) (dir.), Otto Wagner : Reflections on the Raiment of Modernity, Santa Monica, Getty Publications Program, p. 245-278.

BLANC (Charles) [1867], 1880, Grammaire des arts du dessin, Paris, Laurens.

BLANC (Charles), 1882, Grammaire des arts décoratifs, Paris, Loones ( $2^{\mathrm{e}}$ édition, augmentée d'une introduction).

BOURLIER (Pierre), 2006, Style, temps, architecture, ou l'éternel retour de l'enjeu stylistique, Thèse de doctorat, Université Paris 8.

BRESSANI (Martin), 1996, "Opposition et équilibre : le rationalisme organique de Viollet-le-Duc », Revue de l'art, n¹12, p. 28-37.

Chalgnet (Édouard Antelme), 1860, Les Principes de la science du Beau, Paris, Durand.

CHIPIEZ (Charles), 1876, Histoire critique des origines et de la formation des ordres grecs, Paris, Morel.

CLOQUET (Louis), 1894, Essai sur les principes du beau en architecture, Gand, Desclée, de Brouwer et $C^{\text {ie }}$.

CLOQUET (Louis), 1895, Esthétique architecturale. Essai de classification et d'appréciation des formes, Bruxelles, de Polleunis et Ceuterick.

ClOQUET (Louis), 1913, Traité d'architecture. Eléments de l'architecture, type d'édifices, esthétique, composition et pratique de l'architecture, tome cinquième, Esthétique, composition et décoration, Liège, C. Béranger.

ColRat (Jean), 2008, «Eugène Véron : contribution à une histoire de l'esthétique au temps de Spencer et de Monet ", Revue d'histoire des sciences humaines, $n^{\circ} 18$, p. 203-228.

Constant-Dufeux (Simon Claude), 1848, “Discours prononcé par M. Constant-Dufeux le $1^{\text {er }}$ novembre 1844 à l'inauguration du monument de Dumont d'Urville ", Revue générale de l'architecture et des travaux publics, vol. VIII, col. 440-448.

FORTY (Adrian), 2000, "Function », dans Words and Building, a Vocabulary of Modern Architecture, Londres, Reaktion Books, p. 174-195.

GARGIANI (Roberto), 1997, " "Le style" de Gottfried Semper : vers la construction incorporelle », EAV, École d'architecture de Versailles, $\mathrm{n}^{\circ} 4,4^{\mathrm{e}}$ trimestre, p. 70-81.

GUILLERME (André) (dir.), 1987, Amphion, vol.1, Le droguier du fonctionnalisme et vol.2, L'officine du fonctionnalisme, Paris, Picard.

GUYAU (Jean-Marie), 1884, Les Problèmes de l'esthétique contemporaine, Paris, Alcan.

GUYAU (Jean-Marie), 1889, L'Art au point de vue sociologique, Paris, Alcan.

JOEDICKE (Jürgen), 1971, « De la genèse du fonctionnalisme ", L'Architecture d'aujourd'hui, n¹58, p. 18-27.

LAURENT (Stéphane), 1998, L'Art utile: les écoles d'arts appliqués en France (1851-1940), Paris, L'Harmattan. 
LeVEQUE (Charles), 1872, La Science du Beau étudiée dans ses principes, dans ses applications et dans son histoire, Paris, Durand.

MALLGRAVE (Harry F.), 1993, «From Realism to Sachlichkeit : the Polemics of Architectural Modernity in the 1890's ", dans Otto Wagner: Reflections on the Raiment of Modernity, Santa Monica, Getty Publications Program, p. 281-282.

Mallgrave (Harry F.), 1996, Gottfried Semper : Architect of the Nineteenth Century, New Haven, Yale University Press.

MANIERI-EliA (Mario), 1996, "Form Follows Function? » dans Louis Henry Sullivan, New York, Princeton Architectural Press, p. 114-125.

MCWILLIAM (Neil) [1993], 2007, Rêves de bonheur. L'art social dans la gauche française (1830-1950), Dijon, Les Presses du Réel (tr. fr. de Dreams of Happiness, Social Art and the French Left 1830-1850)

REICHLIN (Bruno), 1997, "L'Infortune critique du fonctionnalisme ", dans COHEN (Jean-Louis) (dir.), Les Années 30. L'architecture et les arts de l'espace entre industrie et nostalgie, Paris, Éditions du Patrimoine, p. 186-196.

RICHARDS (James M.), 1958, The Functional Tradition in Early Industrial Buildings, Londres, Architectural Press.

SCHNAIDT (Claude), Gallo (Emmanuelle), 1988, «Qu'est-ce que le fonctionnalisme ? », Archithese, n'18, p. 8-10.

SEMPER (Gottfried), 1860-63, Der Stil in den technischen und tektonischen Künsten oder praktische Ästhetik, Francfort, Verlag für Kunst und Wissenschaft (tr. fr. partielle, 2007, Du style et de l'architecture. Écrits 1834-1869, Marseille, Parenthèses).

SfAELlos (Charambalos) (1952), Le Fonctionnalisme dans l'architecture contemporaine, Paris, Vincent, Fréal et $C^{\text {ie }}$.

SOULILLOU (Jacques), "Introduction ", dans Gottfried Semper, Du style et de l'architecture. Écrits 1834-1869, Marseille, Parenthèses, 2007, p. 7-31.

SOURIAU (Paul), 1904, La Beauté rationnelle, Paris, Alcan.

SPENCER (Herbert), 1877, "L'Utile et le beau ", Essais de morale, de science et d'esthétique. I. Essais sur le progrès, Paris, Germer Baillière et $C^{\text {ie }}$.

StAVRINAKI (Maria), 2008, "L'architecture du pays du milieu: la solution sublime ", introduction à BEHNE (Adolf), La Construction fonctionnelle moderne, Paris, Éditions de la Villette, p. 7-25.

THIBAULt (Estelle), 2010, La Géométrie des émotions. Les esthétiques scientifiques de l'architecture en France, 1860-1950, Wavre, Mardaga.

VAN EYCK (Caroline), 1994, Organicism in Nineteenth Century Architecture: an Inquiry into its Theoretical and Philosophical Background, Amsterdam, Architectura \& Natura Press.

VAN ZANTEN (David), WIT (Wim de), 1986, Louis Sullivan : The Function of Ornament, New York, W. W. Norton.

VentuRI (Robert) [1966], 1996, De l'ambiguïté en architecture, Paris, Dunod (tr. fr. de Complexity and Contradiction in Architecture)

VERON (Eugène), 1878, L'Esthétique : origine des arts, le goût et le génie, définition de l'art et de l'esthétique, le style, l'architecture, la sculpture, la peinture, la danse, la musique, la poésie, Paris, C. Reinwald (rééd. 2007, avec une préface de Jacqueline Lichtenstein).

VIOLLET-LE-DUC (Eugène Emmanuel), Entretiens sur l'architecture, Paris, Morel, 1863-72.

VIOLLET-LE-Duc (Eugène Emmanuel) [1864], 1994, Esthétique appliquée à l'histoire de l'art, Paris, École nationale supérieure des beaux-arts (réédition des cours professés par Viollet-le-Duc en 186364).

ZURCO (Edward de), 1957, The Origins of Functionalist Theory, New York, Columbia University Press. 